\title{
Consumo alimentar de gestantes de alto risco através de um índice de qualidade da
}

\section{dieta adaptado}

\author{
Food consumption of high-risk pregnant women through an adapted diet quality index \\ Consumo alimentar de mujeres embarazadas de alto riesgo a través de un índice de calidad de la \\ dieta adaptado
}

Recebido: 05/03/2021 | Revisado: 09/03/2021 | Aceito: 12/03/2021 | Publicado: 20/03/2021

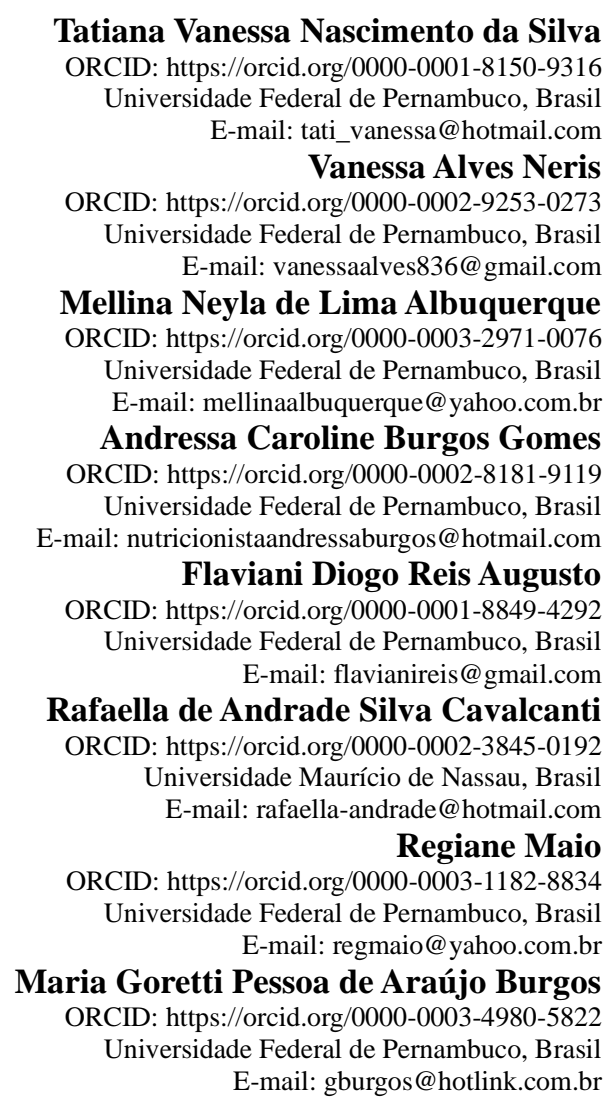

\section{Resumo}

A qualidade da alimentação está relacionada ao ganho de peso gestacional, bem-estar materno e desenvolvimento fetal, sendo assim, o presente trabalho objetivou investigar o índice de qualidade da dieta de gestantes de alto risco. Estudo descritivo, transversal e natureza quantitativa, com gestantes de alto risco acompanhadas no Hospital das Clínicas da Universidade Federal de Pernambuco (HCUFPE), realizado de agosto a novembro de 2020. A amostragem realizada por conveniência sendo incluídas todas gestantes de alto risco elegíveis para pesquisa. Foram coletados dados socioeconômicos, demográficos, clínicos, obstétricos e antropométricos. A ingestão dietética obtida pelo recordatório alimentar de $24 \mathrm{~h}$, cuja análise resultou no índice de qualidade da dieta adaptado para gestantes (IQDAG), onde maiores escores indicam melhor padrão alimentar. Para análise estatística foram utilizados testes Exato de fisher, Qui-Quadrado de Pearson e Kruskal-Wallis, com nível de significância de 5\%. A amostra constituída

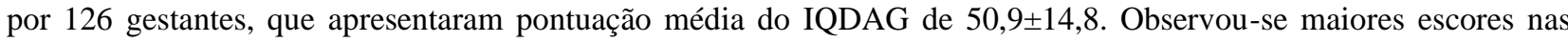
gestantes de maior idade, escolaridade e renda familiar, empregadas, que iniciaram a gestação eutróficas e com síndromes hipertensivas na gestação. Exceto o grupo das leguminosas, a adequação dos demais componentes foi inferior a $50 \%$ na maioria da amostra. A ingestão de ultraprocessados apresentou forte correlação inversa com o IQDAG $(-0,674)$, presente em grande quantidade na dieta das gestantes com menores pontuações. A avaliação da qualidade alimentar pelo IQDAG apresentou alto consumo de ultraprocessados, baixa ingestão alimentar de nutrientes essenciais como folato, ferro, fibra, ômega 3 e porções inferiores ao recomendado de hortaliças e frutas frescas.

Palavras-chave: Gravidez de alto risco; Dieta; Consumo de alimentos; Nutrição pré-natal. 


\begin{abstract}
The quality of dietary intake is related to weight gain during pregnancy, maternal wellbeing and fetal development, therefore, the present study aimed to investigate the diet quality index of high-risk pregnant women. A descriptive, quantitative cross-sectional study carried out with high-risk pregnant women, attended at Hospital das Clínicas of Federal University of Pernambuco (HCUFPE), between August and November 2020. Sampling carried out by convenience, including high-risk pregnant women eligible for research. Information on socioeconomic, demographic, clinical, obstetric and anthropometric data were collected. Dietary intake was obtained from a 24-hour food record, whose analysis resulted in the diet quality index adapted for pregnant women (IQDAG) which higher scores indicate a better dietary pattern. To statistical analysis were used Fisher's exact, Pearson's Qui-Square and Kruskal-Wallis tests, with a significance level of 5\%. The sample was 126 high-risk pregnant women, and the average IQDAG score was $50,9 \pm 14,8$. Better quality of diet was observed among pregnant women of older age, better degree of education, who have participation in the labor Market, who started gestation with adequate weight and diagnosed with Hypertensive Gestational Syndrome. Except the legume group, the adequacy of the other components was lower than 50\% in most of the sample. The intake of ultra-processed foods showed an inverse correlation with IDQAD (-0,674), present in large amounts in the diet of pregnant women with the lowest scores. The IQDAG evaluation revealed an elevated consumption of ultra-processed foods, deficiency of essential nutrients such as folate, iron, fiber, omega-3, and low consumption of vegetables and fresh fruits.
\end{abstract}

Keywords: Pregnancy high-risk; Diet; Food consumption; Prenatal nutrition.

\title{
Resumen
}

La calidad alimentar está relacionada a la ganancia de peso gestacional, bienestar materno y desarollo fetal, siendo así, el objetivo del presente trabajo fue investigar el índice de calidad de la dieta de mujeres embarazadas de alto riesgo. Se trata de un estudio descriptivo, transversal y naturaleza cuantitativa, con embarazadas de alto riesgo acompañadas en el Hospital de las Clínicas de la Universidad Federal de Pernambuco (HCUFPE), realizado de agosto a noviembre de 2020. El muestreo fue hecho por conveniencia incluyéndose todas las embarazadas de alto riesgo elegibles para la encuesta. Fueron recolectados datos sociodemográficos, clínicos, obstétricos y antropométricos. La ingesta dietética fue obtenida por medio del recordatorio de 24 horas cuyo análisis resultó en el índice de calidad de la dieta adaptado para gestantes (IQDAG), en que mayores puntajes indican mejor patrón alimentario. Para el análisis estadístico fueron utilizadas las pruebas Exacta de Fisher, Chi-cuadrada de Pearson y Kruskal-wallis, con un nivel de significación del $5 \%$. La muestra fue constituida por 126 gestantes de alto riesgo, que presentaron puntuación media del IQDAG de $50,9 \pm 14,8$. Se pudo observar mayores puntajes del IQDAG en las gestantes con más edad, escolaridad y renta familiar, incluidas en el mercado de trabajo, que iniciaron la gestación eutróficas y con transtornos hipertensivos en el embarazo. Con excepción de las leguminosas, la adecuación de los demás componentes fue inferior a 50\% en la mayoria de la muestra. La ingestión de ultraprocesados presentó fuerte correlación inversa con el IQDAG (-0,674), presente en gran cantidad en la dieta de gestantes con puntuaciones más bajas. La evaluación de la calidad alimentar por el IQDAG presentó alto consumo de ultraprocesados, baja ingestión alimentar de nutrientes esenciales como folato, hierro, fibra, omega-3 y porciones más pequeñas que las recomendadas de hortalizas y frutas frescas.

Palabras clave: Gestación de alto riesgo; Dieta; Consumo de alimentos; Nutrición prenatal.

\section{Introdução}

A gestação é um processo fisiológico que cursa com alterações estruturais e nas funções orgânicas da mulher, criando um ambiente favorável para a adaptação do organismo e a manutenção da gravidez a fim de garantir o desenvolvimento do feto, da placenta e de outros tecidos maternos (Furlan et al., 2019). Durante esse período, ocorre aumento nas demandas energéticas e de nutrientes essenciais específicos, com o objetivo de suprir as necessidades para o adequado desenvolvimento fetal (Gadelha et al., 2020).

Na maioria das vezes, a gestação cursa sem intercorrências, porém, em $20 \%$ dos casos há a probabilidade de evoluir com alguma complicação, configurando em gestação de alto risco, aumentando as chances de desfechos desfavoráveis para a mãe e o feto (Rodrigues et al., 2017). São vários os fatores preditores de risco na gravidez, com destaque para as síndromes hipertensivas na gestação (SHG) e o Diabetes Mellitus Gestacional (DMG), condições que podem estar relacionadas ou serem agravadas pelo consumo alimentar e estado nutricional inadequados (Avalos et al., 2020; Nogueira et al., 2020).

A mortalidade materna está relacionada à gestações de alto risco. Dados recentes têm evidenciado queda nessas taxas, em torno de $44 \%$ no mundo e de $58 \%$ no Brasil, porém, os índices permanecem preocupantes, principalmente nos países em desenvolvimento (Fernandes et al., 2019; Organização Pan-Americana de Saúde, 2018). 
Registros do Departamento de Informática do Sistema Único de Saúde (DATASUS) revelam uma taxa de 60,10 mortes de mulheres durante a gravidez, parto, aborto ou puerpério a cada 100.000 nascidos vivos entre 2008 e 2014 em Pernambuco, valor considerado elevado pela Organização Mundial da Saúde (OMS) (Ribeiro \& Rocha, 2018).

O processo de transição nutricional vivenciado pela população brasileira nas últimas décadas, caracterizado pelo aumento do uso de alimentos industrializados, ricos em açúcares, sódio, gordura saturada e trans, têm chamado atenção em especial para mulheres em idade fértil, havendo nessa população o aumento do excesso de peso e ganho de peso gestacional acima do recomendado (Santana \& Sarti, 2019; Souza et al., 2019). Mulheres que iniciam a gestação com excesso de peso têm o risco elevado de complicações, semelhante ao risco para aquelas de baixo peso, com frequentes desfechos desfavoráveis para mãe e feto (Catalano \& Shankar, 2017).

Está bem estabelecida a associação entre fatores nutricionais e o desenvolvimento de patologias ou complicações na gestação (Gomes et al., 2019). Tendo em vista a importância de uma ingestão alimentar adequada, a avaliação do consumo alimentar de gestantes é necessária para identificar distúrbios nutricionais e metabólicos (Zuccolotto et al., 2019).

O Índice de Qualidade da Dieta Adaptado para Gestantes (IQDAG), foi desenvolvido para avaliar a qualidade alimentar de gestantes atendidas pelo Sistema Único de Saúde (SUS), no município de Ribeirão Preto em São Paulo. O IQDAG foi baseado no Índice de Qualidade da Dieta Revisado (IQD-R), no Índice de Alimentação Saudável para Gestantes Brasileiras (HELP-B) e nos dez passos da alimentação saudável para gestantes, recomendados pelo Ministério da Saúde em 2013 (Crivellenti et al., 2018).

Como componente moderador da dieta, Crivellenti et al. (2018), incorporou no IQDAG a porcentagem calórica proveniente de alimentos ultraprocessados como bebidas açucaradas, biscoitos, pães industrializados, macarrão instantâneo, sorvetes, chocolates, enlatados, embutidos e produtos congelados prontos para aquecimento, conforme recomendações do guia alimentar para a população brasileira.

Estudos que avaliam aspectos relacionados à qualidade alimentar das gestantes constituem uma ferramenta indispensável para a promoção de ações que ampliem o acesso a um acompanhamento eficaz dessas mulheres, possibilitando intervenções e manejo adequado, promovendo melhores condições de saúde e nutrição materno infantil. Sendo assim, o objetivo do presente estudo foi investigar o índice de qualidade da dieta de gestantes de alto risco.

\section{Metodologia}

Trata-se de um estudo do tipo descritivo, de corte transversal e de natureza quantitativa (Pereira et al., 2018). Realizado de agosto a novembro de 2020, no qual foram avaliadas 126 gestantes de alto risco acompanhadas no centro obstétrico, alojamento conjunto e ambulatório de obstetrícia do Hospital das Clínicas, da Universidade Federal de Pernambuco (HC/UFPE), centro de referência em Pernambuco para gestação de alto risco. A amostragem realizada por conveniência, assim, incluídas todas as gestantes de alto risco que se enquadraram nos critérios de elegibilidade.

Participaram da pesquisa gestantes de alto risco com idade $\geq 19$ anos a partir do segundo trimestre de gravidez. Foram excluídas gestantes com mais de 6 horas de admissão na enfermaria, as que tinham realizado alguma refeição no hospital, gestações múltiplas, pacientes com incapacidade cognitiva para responder o questionário (com problemas psiquiátricos e/ou cognitivos), pacientes submetidas a cirurgias ou que apresentassem patologias prévias que modificassem a composição corporal tais como: hiperêmese gravídica, insuficiência renal aguda ou crônica, hepatopatias e cardiopatias graves. As participantes convidadas receberam um Termo de Consentimento Livre e Esclarecido (TCLE) antes da entrevista e em caso de concordância e assinatura foram incluídas no estudo.

Como prevenção da disseminação do novo coronavírus SARS Cov2, foram adotadas medida de prevenção, como: distanciamento de um metro durante a coleta, o uso de equipamento de proteção individual (EPI) pelo pesquisador e pela 
gestante (máscara), higienização das mão e equipamentos utilizados antes e após o contato.

Os dados sobre a caracterização da população foram coletados através da aplicação de um questionário desenvolvido pelos pesquisadores, estruturado com os dados sociodemográficos (idade, raça, procedência, estado civil, escolaridade, ocupação e renda familiar), antropométricos (peso pré-gestacional, peso atual, índice de massa corporal (IMC) prévio e atual e ganho de peso) obstétricos e clínicos (idade gestacional e patologias associadas a gestação), obtidos por meio de entrevista com a participante, consulta ao prontuário e cartão da gestante.

O estado nutricional prévio foi determinado conforme as recomendações da OMS para pessoas adultas, por meio do cálculo do IMC, utilizando o peso pré-gestacional (Institute of Medicine, 2009). Também foi investigado o ganho de peso gestacional e o estado nutricional atual através do peso mais recente, e classificado de acordo com valores de IMC para a idade gestacional indicados por Atalah et al. (1997).

O IQDAG foi obtido a partir da análise de um Recordatório alimentar de 24 horas (R24h), aplicado no momento da entrevista, no qual a gestante relatou os alimentos e bebidas consumidos nas últimas 24 horas. Para auxílio na determinação da quantidade das porções ingeridas, foi utilizado um álbum com fotos coloridas de utensílios e alimentos, objetivando melhor precisão (Crispim et al., 2017).

Os alimentos foram convertidos em energia, fibra, macro e micronutrientes de interesse para o estudo através do aplicativo Nutrisoft Brazil, com inserção das informações em medidas caseiras (Nutrisoft Brazil, 2021). Para os alimentos não contemplados no referido programa foi consultada a Tabela Brasileira de Composição Química dos Alimentos (2011).

Quadro 1. Componentes e critérios para pontuação do Índice de Qualidade da Dieta Adaptado para Gestantes (IQDAG).

\begin{tabular}{|l|c|c|c|}
\hline \multicolumn{1}{|c|}{ Componente } & \multicolumn{2}{c|}{} \\
\hline Pontuação & $\mathbf{0}$ & $\mathbf{1 0}$ & $\mathbf{2 0}$ \\
\hline Hortaliças/ 1.000 kcal (em porções) & $\mathbf{0}$ & $\geq \mathbf{1 , 5}$ & \\
\hline Leguminosas/ 1.000 kcal (em porções) & $\mathbf{0}$ & $\geq \mathbf{0 , 5}$ & \\
\hline Frutas frescas/ 1.000 kcal (em porções) & $\mathbf{0}$ & $\geq \mathbf{1 , 5}$ & \\
\hline Fibras (g) & $\mathbf{0}$ & $\geq \mathbf{2 8}$ & \\
\hline Ômega 3 (mg) & $\mathbf{0}$ & $\geq \mathbf{1 , 4}$ & \\
\hline Cálcio (mg) & $\mathbf{0}$ & $\geq \mathbf{8 0 0}$ & \\
\hline Folato (mg) & $\mathbf{0}$ & $\geq \mathbf{5 2 0}$ & \\
\hline Ferro (mg) & $\mathbf{0}$ & $\geq \mathbf{2 2}$ & \\
\hline Alimentos ultraprocessados (\%VET) & $\mathbf{2 4 5}$ & & $\leq \mathbf{1 8}$ \\
\hline
\end{tabular}

Fonte: Crivellenti et al. (2018)

No Quadro 1 consta os alimentos e nutrientes avaliados pelo IQDAG. Foram analisados três grupos alimentares (Hortaliças, leguminosas e frutas frescas) e cinco nutrientes (fibras, ômega 3, cálcio, folato e ferro), os quais foram atribuídos pontuação de 0 a 10 conforme adequação do consumo. Os alimentos ultraprocessados foram convertidos em calorias e avaliados de acordo com a participação no valor energético total (VET), pontuando entre 0 e 20, sendo o consumo recomendado inferior a 18\% e acima de 45\% do VET não recebeu pontuação (Brasil, 2014; Brasil, 2013; Crivellenti et al., 2018). 
O IQDAG considerou o número de porções diárias de frutas frescas e hortaliças, recomendadas pelo Ministério da Saúde nos dez passos da alimentação saudável para gestante. As porções foram estimadas a cada 1.000 calorias, sendo 1,5 porções de hortaliças, 1,5 porções de frutas frescas e 0,5 porção de leguminosas (Brasil, 2013; Fisberg et al., 2004).

Para os nutrientes cálcio, folato, ferro e fibras foram utilizado valores de referência para gestante do HELP-B, o ômega 3 foi avaliado considerando a importância desse nutriente para a saúde materna e fetal (Crivellenti et al., 2018; Melere et al., 2013).

$\mathrm{Na}$ análise estatística do IQDAG a população foi dividida em tercis, com pontuações variando de 0 a 100 pontos, quanto maior a pontuação melhor a qualidade alimentar, sendo o primeiro tercil com as menores pontuações e "baixa qualidade da dieta", o segundo tercil com as pontuações intermediárias com dieta "precisando de melhorias" e o tercil com as maiores pontuações "dieta de boa qualidade".

Os resultados descritivos foram expressos em frequências absolutas e percentuais. Para avaliar diferença entre os percentuais relativos às categorias de uma variável foi utilizado o teste Qui-quadrado de Pearson para igualdade de proporções. No estudo da associação entre duas variáveis categóricas foi utilizado o teste Qui-quadrado de Pearson ou o Exato de Fisher quando a condição para utilização do teste Qui-quadrado não foi verificada. Na comparação entre categorias em relação a variável numérica o teste Kruskal-Walis.

Para avaliar o grau da relação entre duas variáveis numéricas foi obtido o coeficiente de correlação de Pearson ou de Spearman. O nível de significância utilizado na decisão dos testes estatísticos foi de 5\%. Os intervalos foram obtidos com 95\% de confiança. Os dados foram digitados na planilha Excel e o programa utilizado para obtenção dos cálculos estatísticos foi o Statistical Package For The Social Sciences (SPSS) na versão 25.

A pesquisa foi realizada após aprovação pelo Comitê de Ética em Pesquisa, do HC/UFPE, sob o CAEE 26961019.2.0000.8807, em obediência à Resolução 466/12 sobre "Pesquisa Envolvendo Seres Humanos", do Conselho de Saúde do Ministério da Saúde.

\section{Resultados}

Participaram do estudo 126 gestantes de alto risco, com idade média de $29( \pm 6,58)$ anos variando entre 19 e 45 anos e idade gestacional de $31( \pm 6,33)$ semanas, $84,9 \%$ tinham companheiro (casadas/união estável), sendo a maioria da região metropolitana do Recife (58,7\%), não branca $(77,8 \%)$, com mais de 8 anos de estudo (71,4\%), desempregadas $(64,1 \%)$ e com renda familiar superior a 1 salário mínimo $(58,7 \%)$.

Em relação as variáveis clínicas, o principal diagnóstico foi síndromes hipertensivas na gestação (SHG) (53,2\%), seguido de DMG, insuficiência cervical, asma, infecção do trato urinário (ITU), lúpus e cisto no ovário. Houve uma frequência de 95,2\% em relação ao uso de suplementos medicamentosos a base de ácido fólico, sulfato ferroso e polivitamínico. No atual estudo não foi observada correlação significativa entre as variáveis sóciodemográficas, antropométricas, clínicas e obstétricas com a pontuação do IQDAG.

Nesse estudo a prática de atividade física não sofreu tratamento estatístico, pois aquelas que o faziam previamente suspenderam na gestação, pela necessidade de isolamento social, como medida preventiva durante a pandemia.

$\mathrm{Na}$ avaliação do estado nutricional, o ganho de peso gestacional foi de $9,26 \pm 7,17 \mathrm{~kg}$, com $65,1 \%$ da amostra tendo iniciado a gestação com peso acima do recomendado e $73 \%$ apresentaram excesso de peso, segundo o IMC gestacional.

A média de pontuação do IQDAG aplicado para avaliar a qualidade da dieta foi de 50,9 $( \pm 14,8)$, variando entre 14,2 a 82,5 pontos. $38 \%$ das gestantes consumiam um padrão alimentar reduzido em ultraprocessados ( $<$ de $18 \%$ do VET); consumo adequado foi observado em 27,7\% $(\mathrm{n}=35)$ para frutas frescas; $11,1 \%(\mathrm{n}=14)$ para hortaliças; $63,5 \%(\mathrm{n}=80)$ para leguminosas; $27,7 \%(\mathrm{n}=35)$ para fibras alimentares; 5,5\% $(\mathrm{n}=7)$ para ômega $3 ; 1,5 \%(\mathrm{n}=2)$ para folato; $36,5 \%(\mathrm{n}=46)$ para cálcio e $6,3 \%(\mathrm{n}=$ 
8) para ferro.

$\mathrm{Na}$ análise da qualidade alimentar pelo IQDAG, foi possível observar que apesar de não estar associado de forma significativa com o estado nutricional pré-gestacional e atual, mulheres que iniciaram a gravidez com peso inadequado, apresentaram escores mais baixos em relação as que tiveram os maiores escores. Ao mesmo tempo, foi possível identificar que as gestantes que apresentaram maior pontuação tinham idade mais elevada, maior escolaridade e renda familiar, estavam inseridas no mercado de trabalho, eram eutróficas e possuíam diagnóstico clínico de SHG, em relação as de menor pontuação no IQDAG (Tabela 1).

Tabela 1. Características sociodemográficas, clínicas, obstétricas e antropométricas, segundo o Índice de Qualidade da Dieta Adaptado para Gestantes. HC/UFPE, Recife - PE, 2020.

\begin{tabular}{|c|c|c|c|c|}
\hline Variável & $\begin{array}{c}1^{\circ} \text { Tercil } \\
n=42\end{array}$ & $\begin{array}{c}2^{\circ} \text { Tercil } \\
n=42\end{array}$ & $\begin{array}{c}3^{\circ} \text { Tercil } \\
n=42\end{array}$ & Valor de $p$ \\
\hline Pontuação (mín.; máx.) & $(14,2 ; 44,8)$ & $(45,3 ; 58,2)$ & $(58,4 ; 82,5)$ & \\
\hline Idade média (DP) & $22,1(6,1)$ & $29,3(7,3)$ & $31,1(6,0)$ & $0,334^{(1)}$ \\
\hline 19 a 24 & $13(35,1)$ & $15(40,5)$ & $9(24,3)$ & \\
\hline 25 a 29 & $11(47,8)$ & $6(26,1)$ & $6(26,1)$ & \\
\hline 30 a 34 & $10(30,3)$ & $10(30,3)$ & $13(39,4)$ & \\
\hline 35 a 45 & $8(24,2)$ & $11(33,3)$ & $14(42,4)$ & \\
\hline Raça & & & & $0,418^{(1)}$ \\
\hline Branca & $7(25,0)$ & $12(42,9)$ & $9(32,1)$ & \\
\hline Não branca & $35(35,7)$ & $30(30,6)$ & $33(33,7)$ & \\
\hline Estado civil & & & & $0,648^{(1)}$ \\
\hline Casada & $34(31,8)$ & $37(34,6)$ & $36(33,6)$ & \\
\hline Solteira & $8(42,1)$ & $5(26,3)$ & $6(31,6)$ & \\
\hline Escolaridade & & & & $0,350^{(1)}$ \\
\hline$\geq 8$ anos de estudos & $30(33,3)$ & $27(30,0)$ & $33(36,7)$ & \\
\hline$<8$ anos de estudos & $12(33,3)$ & $15(41,7)$ & $9(25,0)$ & \\
\hline Ocupação & & & & $0,376^{(1)}$ \\
\hline Trabalha & $12(27,3)$ & $14(31,8)$ & $18(40,9)$ & \\
\hline Não trabalha & $30(36,6)$ & $28(34,1)$ & $24(29,3)$ & \\
\hline Renda familiar $^{(3)}$ & & & & $0,201^{(1)}$ \\
\hline$\geq 1$ salário & $24(32,4)$ & $21(28,4)$ & $29(39,2)$ & \\
\hline$<1$ salário & $18(34,6)$ & $21(40,4)$ & $13(25,0)$ & \\
\hline Procedência & & & & $0,795^{(1)}$ \\
\hline Recife e região metropolitana & $23(31,1)$ & $26(35,1)$ & $25(33,8)$ & \\
\hline Interior de PE & $19(36,5)$ & $16(30,8)$ & $17(32,7)$ & \\
\hline Trimestre gestacional & & & & $0,458^{(1)}$ \\
\hline Segundo trimestre & $27(31,0)$ & $32(36,8)$ & $28(32,2)$ & \\
\hline Terceiro trimestre & $15(38,5)$ & $10(25,6)$ & $14(35,9)$ & \\
\hline Patologia & & & & $0,838^{(1)}$ \\
\hline DMG & $10(34,5)$ & $10(34,5)$ & $9(31,0)$ & \\
\hline SHG & $22(32,8)$ & $20(29,9)$ & $25(37,3)$ & \\
\hline Outras comorbidades ${ }^{(4)}$ & $10(33,3)$ & $12(40,0)$ & $8(26,7)$ & \\
\hline Suplementos medicamentosos ${ }^{(5)}$ & & & & $0,365^{(2)}$ \\
\hline Sim & $41(34,2)$ & $38(31,7)$ & $41(34,2)$ & \\
\hline Não & $1(16,7)$ & $4(66,7)$ & $1(16,7)$ & \\
\hline IMC pré-gestacional & & & & $0,414^{(2)}$ \\
\hline Baixo peso & $1(12,5)$ & $5(62,5)$ & $2(25,0)$ & \\
\hline Adequado & $9(25,0)$ & $12(33,3)$ & $15(41,7)$ & \\
\hline
\end{tabular}




\begin{tabular}{|c|c|c|c|c|}
\hline $\begin{array}{l}\text { Sobrepeso } \\
\text { Obesidade }\end{array}$ & $\begin{array}{l}12(34,3) \\
20(42,6)\end{array}$ & $\begin{array}{l}11(31,4) \\
14(29,8)\end{array}$ & $\begin{array}{l}12(34,3) \\
13(27,7)\end{array}$ & \\
\hline IMC atual & & & & $0,864^{(2)}$ \\
\hline Baixo peso & $3(25,0)$ & $6(50,0)$ & $3(25,0)$ & \\
\hline Adequado & $6(27,3)$ & $8(36,4)$ & $8(36,4)$ & \\
\hline Sobrepeso & $13(40,6)$ & $9(28,1)$ & $10(31,3)$ & \\
\hline Obesidade & $20(33,3)$ & $19(31,7)$ & $21(35,0)$ & \\
\hline
\end{tabular}

IMC: índice de massa corporal. SHG: síndromes hipertensivas na gestação. DMG: diabetes mellitus gestacional. (1) Através do teste Qui-quadrado de Pearson. (2) Pelo teste Exato de Fisher. (3) Valor em $2020.2=$ R\$ 1.045. (4) Insuficiência cervical, asma, infecção do trato urinário (ITU), lúpus, e cisto no ovário. (5) Ácido fólico, sulfato ferroso e polivitamínicos. Fonte: Autores.

Exceto para o ômega 3, os demais componentes (alimentos e nutrientes) do IQDAG, apresentaram correlação estatisticamente significativa com a pontuação final (Tabela 2). O consumo de ultraprocessados apresentou forte correlação inversa com a pontuação final do índice, ou seja, quanto maior o consumo de ultraprocessados menor o escore do IQDAG (Tabela 2).

Tabela 2. Mediana (P25; P75) do consumo em porções dos alimentos e valores dos nutrientes, do Índice de qualidade da Dieta Adaptado para Gestantes e sua correlação com a pontuação final. HC/UFPE, Recife PE, 2020.

\begin{tabular}{lccc} 
Componente & Mediana (P25; P75) & $(\mathbf{r})$ & $(\mathbf{p})$ \\
\hline Alimentos ultraprocessados(\%VET) & $23,9(14,7 ; 36,9)$ & $-0,674^{(1)}$ & $<0,001^{*}$ \\
Frutas frescas/ 1.000 kcal (em porções) & $0,4(0,0 ; 1,4)$ & $0,464^{(1)}$ & $<0,001^{*}$ \\
Hortaliças/ 1.000 kcal (em porções) & $0,0(0,0 ; 0,7)$ & $0,350^{(1)}$ & $<0,001^{*}$ \\
Leguminosas/ 1.000 kcal (em porções) & $0,5(0,2 ; 0,7)$ & $0,256^{(1)}$ & $0,004^{*}$ \\
Fibra (g) & $22,2(12,3 ; 21,8)$ & $0,477^{(2)}$ & $<0,001^{*}$ \\
Folato ( $\mu \mathrm{g})$ & $154,7(74,7 ; 267,4)$ & $0,306^{(1)}$ & $<0,001^{*}$ \\
Cálcio (mg) & $624,1(339,0 ; 948,0)$ & $0,401^{(1)}$ & $<0,001^{*}$ \\
Ferro (mg) & $11,6(8,1 ; 15,0)$ & $0,391^{(1)}$ & $<0,001^{*}$ \\
Ômega 3 (mg) & $0,0(0,0 ; 0,0)$ & $0,149^{(1)}$ & 0,097 \\
\hline
\end{tabular}

VET: valor energético total. (*) Estatisticamente diferente de zero a 5\%. (1) Correlação de Spearman. (2) Correlação de Pearson. Fonte: Autores.

A Tabela 3 apresenta a mediana (P25; P75) do consumo de alimentos e nutrientes do IQDAG de cada tercil e a associação com a pontuação final do índice. Foi encontrada associação estatisticamente significativa entre a qualidade da dieta e os componentes do índice, exceto para os alimentos do grupo das leguminosas. 
Tabela 3. Mediana (P25; P75) do consumo dos grupos de alimentos e nutrientes do Índice de Qualidade da Dieta Adaptado para Gestantes (IQDAG) dos tercis e a correlação entre as dietas. HC/UFPE, Recife - PE, 2020.

\begin{tabular}{|c|c|c|c|c|}
\hline \multirow[b]{2}{*}{ Variável } & \multicolumn{3}{|c|}{ IQDAG } & \multirow[b]{2}{*}{ Valor de $p$} \\
\hline & $\begin{array}{c}\mathbf{1}^{\circ} \text { Tercil } \\
(\mathrm{n}=42)\end{array}$ & $\begin{array}{c}2^{\circ} \text { Tercil } \\
(n=42)\end{array}$ & $\begin{array}{c}3^{\circ} \text { Tercil } \\
(n=42)\end{array}$ & \\
\hline $\begin{array}{l}\text { Alimentos } \\
\text { ultraprocessados }\end{array}$ & $\begin{array}{c}39,4 \\
(28,8 ; 52,1)\end{array}$ & $\begin{array}{c}23,9 \\
(15,0 ; 33,5)\end{array}$ & $\begin{array}{c}16,0 \\
(8,3 ; 18,4)\end{array}$ & $<0,001 *$ \\
\hline Frutas & $\begin{array}{c}0,0 \\
(0,0 ; 0,4)\end{array}$ & $\begin{array}{c}0,5 \\
(0,0 ; 1,4)\end{array}$ & $\begin{array}{c}1,2 \\
(0,0 ; 2,3)\end{array}$ & $<0,001 *$ \\
\hline Hortaliças & $\begin{array}{c}0,0 \\
(0,0 ; 0,4)\end{array}$ & $\begin{array}{c}0,0 \\
(0,0 ; 0,7)\end{array}$ & $\begin{array}{c}0,4 \\
(0,0 ; 1,4)\end{array}$ & $0,002^{*}$ \\
\hline Leguminosas & $\begin{array}{c}0,4 \\
(0,0 ; 0,6)\end{array}$ & $\begin{array}{c}0,5 \\
(0,0 ; 0,8)\end{array}$ & $\begin{array}{c}0,6 \\
(0,4 ; 0,8)\end{array}$ & 0,050 \\
\hline Fibras & $\begin{array}{c}16,4 \\
(9,9 ; 21.4)\end{array}$ & $\begin{array}{c}23,6 \\
(18,1 ; 29,8)\end{array}$ & $\begin{array}{c}26,2 \\
(21,3 ; 31,3)\end{array}$ & $<0,001 *$ \\
\hline Folato & $\begin{array}{c}97,3 \\
(36,8 ; 182,5)\end{array}$ & $\begin{array}{c}154,4 \\
(74,6 ; 276,0)\end{array}$ & $\begin{array}{c}173,2 \\
(137,1 ; 304,1)\end{array}$ & $0,002 *$ \\
\hline Cálcio & $\begin{array}{c}467,6 \\
(182,4 ; 703,6)\end{array}$ & $\begin{array}{c}587,9 \\
(341,0 ; 961,5)\end{array}$ & $\begin{array}{c}842,2 \\
(536,5 ; 1100,0)\end{array}$ & $<0,001 *$ \\
\hline Ferro & $\begin{array}{c}9,5 \\
(5,9 ; 11,9)\end{array}$ & $\begin{array}{c}12,8 \\
(8,1 ; 15,1)\end{array}$ & $\begin{array}{c}13,5 \\
(10,4 ; 18,2)\end{array}$ & $<0,001 *$ \\
\hline W3 & $\begin{array}{c}0,0 \\
(0,0 ; 0,0)\end{array}$ & $\begin{array}{c}0,0 \\
(0,0 ; 0,0)\end{array}$ & $\begin{array}{c}0,0 \\
(0,0 ; 0,0)\end{array}$ & $0,038^{*}$ \\
\hline
\end{tabular}

(*) Diferença significativa ao nível de 5,0\%. Valor de $\mathrm{p}=$ pelo teste Kruskal Wallis com comparações do referido teste. Fonte: Autores.

\section{Discussão}

No presente estudo foi avaliado o índice de qualidade da dieta de gestantes de alto risco, acompanhadas em um serviço de referência de Pernambuco. Os resultados encontrados indicaram pontuação do IQDAG $(50,9 \pm 14,8)$ evidenciando qualidade alimentar inferior ao achado por Crivellenti et al. (2018), em estudo realizado para o desenvolvimento do IQDAG

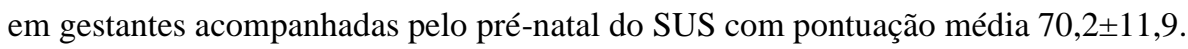

De modo semelhante a esse estudo Crivellenti et al. (2018) não encontraram associação entre características sociodemográficas, clínicas e antropométricas, e o IQDAG. Ao mesmo tempo, a população foi predominantemente de mulheres casadas e com escolaridade acima de 8 anos, corroborando com o encontrado nesta pesquisa.

Kunzler et al. (2020) no Rio Grande do Sul, analisando gestantes de alto risco acompanhadas ambulatorialmente, em que houve predomínio de mulheres com DMG (56,2\%) e SHG (31,3\%), detectaram baixa ingestão de fibras, 19,3g em média, inferior ao encontrado nesta pesquisa com $22,2 \mathrm{~g}$. Ainda, segundo Kunzler, o consumo inadequado de fibras associado à elevada ingestão de carboidratos simples e sódio dificultam o bom controle da DMG e SHG.

Pesquisa em pré-natal no SUS de São Paulo (Crivellenti et al., 2019), encontrou menor qualidade da dieta naquelas 
com excesso de peso gestacional. O mesmo ocorreu em nosso estudo, no qual aquelas no tercil com os menores escores, iniciaram a gravidez com excesso de peso, quando comparado com as que tiveram maior adequação dos componentes da dieta.

Vieira et al. (2020) aplicando o IQDAG, encontraram baixo consumo de frutas frescas e hortaliças por gestantes adolescentes, acompanhadas em unidades básicas de saúde do Rio de Janeiro, onde apenas 19\% do grupo, atingiu as recomendações. De modo diferente, o presente estudo detectou número mais elevado de gestantes consumindo porções adequadas de frutas frescas $(27,7 \%)$, enquanto para uso das hortaliças apenas 11,1\% alcançou o adequado. Ao mesmo tempo, em que no tercil com menor pontuação não houve mediana de consumo desses dois grupos alimentares.

Pesquisas utilizando o IQDAG observaram que o uso de leguminosas por gestantes adultas e adolescentes no Sudeste do Brasil por foi adequado em mais de 50\% da amostra (Crivellenti et al., 2018; Vieira et al., 2020), em concordância com nosso resultado, que teve nas leguminosas maior adequação (63,5\%). O feijão foi o alimento que mais representou esse grupo, que associado com o arroz no almoço, faz parte do padrão alimentar nordestino (Pires \& Gonçalves, 2021).

Em relação a ingestão de nutrientes específicos como ferro, folato, cálcio, ômega 3 e fibra, o consumo foi insuficiente na alimentação da maioria das gestantes desse estudo. No Brasil, a suplementação do ferro e do ácido fólico na gestação são estratégia do Ministério da Saúde no combate à deficiência geralmente encontrada desses nutrientes essenciais para formação e desenvolvimento do feto, justificando o seu uso regular por 95,2\% das gestantes (Brasil. Programa nacional de suplementação de ferro, 2013).

Quanto à ingestão de cálcio, apenas os estudos de Vieira et al. (2020) e Crivellenti et al. (2018), referem valores de $7,1 \%$ e $13,6 \%$ respectivamente, indo de encontro ao consumo detectado em nossas gestantes de 36,5\%, podendo ser explicado provavelmente pelo modismo atual em retirar laticínios da alimentação, muito presente em adolescentes e adultos jovens (Gomes et al., 2019).

O IQDAG introduziu o componente "alimentos ultraprocessados", considerando as recomendações do guia alimentar para a população brasileira. Está bem estabelecido na literatura, que o consumo em excesso desses alimentos está associado ao aumento da obesidade e do desenvolvimento de doenças crônicas não transmissíveis. Em gestantes, o consumo deste grupo de alimentos vem sendo associado ao ganho de peso excessivo e desenvolvimentos de agravos durante a gestação e parto. Além disso, o consumo de alimentos ultraprocessados impacta negativamente na ingestão de micronutrientes essenciais nesse período (Gomes et al., 2020).

Paulino et al. (2020) examinaram o grau de processamento dos alimentos consumidos por gestantes de alto risco, identificaram que 25\% do VET diário era proveniente de ultraprocessados. Resultado semelhante ao de Becker et al. (2020) em coorte observacional com 303 gestantes, com ingestão 26,8\% deste grupo de alimentos, corroborando com o atual estudo em que apresentou mediana de consumo de 23,9\%. Cabe ressaltar que, apenas 38\% de toda amostra consumiu ultraprocessados dentro dos valores aceitáveis de acordo com o IQDAG.

Estudo realizado no Sudeste do Brasil, em que foi avaliado o grau de processamento dos alimentos consumidos em período gestacional (Naspolini et al., 2020), revelou frequência elevada na ingestão de fast-food, comidas embaladas e embutidos, enquanto apenas $8 \%$ relatou uso regular dos alimentos in-natura e minimamente processados. Estes resultados corroboram com nossos achados, que evidenciaram correlação negativa entre a pontuação máxima do IQDAG e consumo de alimentos ultraprocessados.

Como limitações deste estudo, pode-se considerar o reduzido número amostral em decorrência da suspensão das pesquisas devido ao momento de pandemia pelo novo coronavírus SARS CoV2; pouca literatura científica acerca da qualidade da dieta em gestantes utilizando o IQDAG e a fragilidade do método de investigação de consumo alimentar, o R24h, que depende da memória do entrevistado podendo ocorrer subestimação dos alimentos consumidos. 


\section{Considerações Finais}

O padrão alimentar da população estudada pelo IQDAG demonstrou baixa qualidade na maior parte das gestantes estudadas, indicando elevado consumo de alimentos industrializados e de baixa qualidade nutricional, deficiência no consumo nutrientes essenciais como o folato, ferro, fibra, ômega 3 e baixo consumo de hortaliças e frutas frescas.

Apesar das limitações apresentadas, os resultados desta pesquisa reforçam a importância da aplicação de instrumentos que avaliem a qualidade alimentar de gestantes de alto risco, uma vez que conhecendo as principais deficiências alimentares desta população, é possível direcionar ações mais específicas, como educação nutricional desde o início da gestação, com foco na mudança do comportamento alimentar dessas mulheres, para redução de agravos na saúde materno-fetal. Ressalta-se a necessidade de mais estudos voltados para a presente temática, com amostras mais significativas.

\section{Referências}

Atalah, S. E., Castilho, C. L., Castro, R. S. \& Aldea, P. A. (1997). Propuesta de nuevo estándar de evaluácion nutricional em embarazadas. Revista Médica de Chile, 125(12),1429-36. https://pesquisa.bvsalud.org/portal/resource/pt/lil-210390

Avalos, L. A., Caan, B., Nance, N., Zhu, Y., Li, D., Quesenberry, C., Hyde, R. J. \& Hedderson, M. M. (2020). Prenatal Depression and Diet Quality During Pregnancy. Journal Of The Academy Of Nutrition And Dietetics, 120(6), 972-84. https://doi.org/10.1016/j.jand.2019.12.011

Becker, P. C., Mariot, M. D. M., Kretzer, D. C., Bosa, V. L., Goldani, M. Z., Silva, C. H. \& Bernardi, J. R (2020). Can the pregnant woman's food intake be influenced by her clinical condition during pregnancy? Revista Brasileira de Saúde Materno Infantil, 20(2), 515-24. https://doi.org/10.1590/180693042020000200011

Brasil. (2014). Ministério da Saúde. Guia alimentar para a população brasileira. (2a ed.), Brasília (DF): Ministério da Saúde. https://bvsms.saude.gov.br/bvs /publicacoes/guia_alimentar_populacao_brasileira_2ed.pdf

Brasil (2013). Ministério da Saúde. Atenção ao pré-natal de baixo risco. rev. Brasília (DF): Ministério da Saúde. http://bvsms.saude.gov.br/bvs/ publicacoes/atencao_pre_natal_baixo_risco.pdf

Brasil (2013). Ministério da Saúde. Programa Nacional de Suplementação de Ferro: manual de condutas gerais. Brasília (DF): Ministério da Saúde. http://bvsms.saude.gov.br/bvs/publicacoes/manual_suplementacao_ferro_condutas_gerais.pdf

Catalano, P. M \& Shankar, K. (2017). Obesity and pregnancy: mechanisms of short term and long term adverse consequences for mother and child. Bmj, 356(1), 1-16. https://doi.org/10.1136/bmj.j1

Crispim, S. P., Fisberg, R. M., Almeida, C. C. B., Nicolas, G., Knaze, V., Pereira, R. A., Marchiori, D. M. L., Santos, N. A. Steluti, J. \& Slimani, N. (2017). Manual fotográfico de quantificação alimentar - Curitiba (SP): Universidade Federal do Paraná. 147 p. http://www.ucv.edu.br/biblioteca/livro-virtual/manualfotografico-quantificacao-alimentar.pdf

Crivellenti, L. C., Zuccolotto, D. C.C. \& Sartorelli, D. S. (2018). Development of a Diet Quality Index Adapted for Pregnant Women. Revista de Saúde Pública, 52, 59. https://doi.org/10.11606/s1518-8787.2018052000184

Crivellenti, L. C., Zuccolotto, D. C. C., Sartorelli, D. S. (2019). Association between the Diet Quality Index Adapted for Pregnant Women (IQDAG) and excess maternal body weight. Revista Brasileira de Saúde Materno Infantil, 19(2), 275-83. https://doi.org/10.1590/1806-93042019000200002

Fernandes, D. C., Carreno, I., Silva, A. A., Guerra, T. B. \& Adami, F. S. (2019). Relationship between pre gestational nutritional status and type of processing of foods consumed by high-risk pregnant women. Revista Brasileira de Saúde Materno Infantil, 19(2), 351-61. https://doi.org/10.1590/180693042019000200006

Fisberg, R. M., Slater, B., Barros, R. R., Lima, F. D., Cesar, C. L. G., Carandina, L., Barros, M. B. A. \& Goldbaum, M (2004). Índice de Qualidade da Dieta: avaliação da adaptação e aplicabilidade. Revista de Nutrição, 17(3), 301-18. https://doi.org/10.1590/S1415-52732004000300003

Furlan, C., Carli, G. \& Kümpel, D. A. (2019). Excesso de peso e consumo alimentar de gestantes atendidas em unidades básicas de saúde. Saúde (Santa Maria), 45(2), 12-24. https://doi.org/10.5902/2236583436625

Gadelha, I. P., Aquino, P. S., Balsells, M. M. D., Diniz, F. F., Pinheiro, A. K. B., Ribeiro, S. G. \& Castro, R. C. M. B. (2020). Quality of life of high risk pregnant women during prenatal care. Revista Brasileira de Enfermagem, 73(5), 1-7. https://doi.org/10.1590/0034-7167-2019-0595

Gomes, C. B., Malta, M. B., Benício, M. H. D. \& Carvalhaes, M. A. B. L. (2020). Consumption of ultra-processed foods in the third gestational trimester and increased weight gain: a brazilian cohort study. Public Health Nutrition, 1-9. https://doi.org/10.1017 / S1368980020001883

Gomes, C. B., Vasconcelos, L. G., Cintra, R. M. G. C., Dias, L. C. G. D. \& Carvalhaes, M. A. B. L. (2019). Hábitos alimentares das gestantes brasileiras: revisão integrativa da literatura. Ciência \& Saúde Coletiva, 24(6), 2293-306. https://doi.org/10.1590/1413-81232018246.14702017

Institute of Medicine (2009). Weight gain during pregnancy: reexamining the guidelines. The National Academies. Press National research council (US). https://doi.org/10.17226/12584 
Kunzler, D. J., Carreno, I., Silva, A. A., Guerra, T. B., Fassina, P. \& Adami, F. S. (2020). Consumo dietético e estado nutricional pré- gestacional de gestantes de alto risco. Brazilian Journal Of Health Review, 3(4), 8539-54. https://doi.org/10.34119/bjhrv3n4-105

Melere, C., Hoffmann, J. F., Nunes, M. A. A., Drehmer, M. B. C., Ozcariz, S. G. I., Soares, R. M., Manzolli, P. P., Duncan, B. B. \& Camey, S. A. (2013). Índice de alimentação saudável para gestantes: adaptação para uso em gestantes brasileiras. Revista de Saúde Pública, 47(1), 20-28. https://doi.org/10.1590/S0034-89102013000100004

Naspolini, N. F., Machado, P. P., Fróes-asmus, C. I. R., Câmara, V. M., Moreira, J. C. \& Meyer, A. (2020). Food consumption according to the degree of processing, dietary diversity and socio-demographic factors among pregnant women in Rio de Janeiro, Brazil: the rio birth cohort study of environmental exposure and childhood development (pipa project). Nutrition And Health, 26-34.https://doi.org/10.1177/0260106020960881

Nogueira, M. D. A., Santos, C. C., Lima, A. M., Lima, M. R. S., Sousa, F. I. S., Vieira, Li. C. O., Braga, R. A. M. \& Cruz, I. F. S. (2020). Associação entre estado nutricional, diabetes gestacional e doenças hipertensivas em gestantes de risco. Brazilian Journal Of Development, 6(2), 8005-18. https://doi.org/10.34117/bjdv6n2-200

Nutrisoft Brazil (2021). Editora Abril. https://nutrisoft.com.br

Organização pan-americana da saúde (2018). Folha informativa sobre mortalidade materna. Brasília DF. https://www.paho.org/bra/index.php?option $=$ comcontent $\&$ view $=$ article $\&$ id $=5741$ : folha-informativa-mortalidade materna $\&$ Itemid $=820$

Paulino, D. S. M., Pinho-pompeu, M., Assumpção, D., Kasawara, K. T. \& Surita, F. G. (2020). Dietary intake profile in high-risk pregnant women according to the degree of food processing. The Journal Of Maternal-Fetal \& Neonatal Medicine, 1-7. https://doi.org/10.1080 / 14767058.2020.1818213

Pereira, A. S., Shitsuka, D. M., Parreira, F. J. \& Shitsuka, R. (2018). Metodologia da pesquisa científica. UFSM. https://www.ufsm.br/app/uploads/sites/358/2019/02/Metodologia-da-Pesquisa-Cientifica_final.pdf

Pires, I. G. \& Gonçalves D. R. (2021). Consumo alimentar e ganho de peso de gestantes assistidas em unidades básicas de saúde. Brazilian Journal of Health Review, 4(1) 2021, 128-46. https://doi.org/10.34119/bjhrv4n1-013

Ribeiro, R. F. S. A. \& Rocha, E. C. (2018). Redução da mortalidade materna em Pernambuco: realidade ou desafio? Espaço público, 2, 120-34. https://repositorio.ufpe.br/handle/123456789/32326

Rodrigues, A. R. M., Dantas, S. L. C., Pereira, A. M. M., Silveira, M. A. M. \& Rodrigues, D. P. (2017). Gravidez de alto risco: análise dos determinantes de saúde. Sanare, 16(1), 23 - 28. https://sanare.emnuvens.com.br/sanare/article/view/1135/620

Santana, A. B. C. \& Sarti, F. M. (2019). Mapeamento da qualidade nutricional da alimentação em diferentes estados do Brasil. Confins, 39, 1-14. https://journals.openedition.org/confins/18449\#: :text=Em\%20termos\%20geogr\%C3\%A1ficos\%2C\%20identificou\%2Dse,Maranh\%C3\%A3o\%20(60\%2C31)

Souza, J. P. M., Lima, M. M. \& Horta, P. M. (2019). Diet quality among the brazilian population and associated socioeconomic and demographic factors: analysis from the national dietary survey 2008-2009. Journal Of The Academy Of Nutrition And Dietetics, 119(11), 1866-74. https://doi.org/10.1016 / j.jand.2019.04.014

Tabela brasileira de composição de alimentos. (2011). (4a ed.), UNICAMP, 161 p. http://www.nepa.unicamp.br/taco/contar/taco_4_ edicao_ampliada _e_revisada.pdf? arquivo=taco_4_versao_ampliada_e_revisada.pdf

Vieira, M. A., Sally, E. O. F., Barbosa, R. M. S. \& Ferreira, D. M. (2020). Qualidade da dieta de gestantes adolescentes assistidas na Rede Básica de Saúde. Saúde e Pesquisa, 13(3), 515-22. https://doi.org/10.17765/2176-9206.2020v13n3p515-522

Zuccolotto, D. C. C., Crivellenti, L. C., Franco, L. J. \& Sartotelli, D. S. (2019). Dietary patterns of pregnant women, maternal excessive body weight and gestational diabetes. Revista de Saúde Pública, 53, 52-59. https://doi.org/10.11606/s1518-8787.2019053000909 